\title{
Relational variants of categories of fuzzy sets defined by monads
}

\author{
Jiří Močkoř \\ Institute for Research and Applications of Fuzzy Modeling, \\ University of Ostrava, Centre of Excellence IT4Innovations \\ 30. dubna 22, 70103 Ostrava 1, \\ Czech Republic, \\ Jiri.Mockor@osu.cz
}

\begin{abstract}
By a $\mathbf{T}$-relational variant of a category $\mathbf{K}$ we understand a Kleisli category $\mathbf{K}_{\mathbf{T}}$ of a category $\mathbf{K}$ with respect to a monad $\mathbf{T}$. Using explicit definitions we equivalently describe several T-relational examples of categories with fuzzy type objects, where $\mathbf{T}$ are appropriate monads in these categories.
\end{abstract}

Keywords: Residuated lattices, fuzzy relations, categories with fuzzy objects, monads, Kleisli categories

\section{Introduction}

Fuzzy set theory was introduced by Zadeh [30] as a generalization of the classical set theory, allowing working with vagueness, as one of the basic features of real-world applications. Concurrently with its origins, the theory of fuzzy sets dealt not only with objects, i.e., with fuzzy sets, but also investigated the functional relations between these objects. This naturally led to research into the categorical aspects of fuzzy sets and, in general, to exploration of fuzzy set categories. The fuzzy set categories were often designed to be as close as possible to the classical category of sets. This led to an effort to create such categories of fuzzy sets that would be topos $[1,7,25,26,27]$. With the development of the fuzzy set theory and applications, the Heyting algebra was gradually abolished as an array of fuzzy set values and replaced by other complete lattice structures, such as the totally monoidal sets and various generalizations of these structures (see $[3,4,5,6,7,19,20,22,23,24]$ and others). However, it was still valid that the key role of morphisms in these new categories had two main structures, namely the mappings between underlying sets of fuzzy sets with specific properties on the one hand and the fuzzy relation (again with special properties) on the other.
Recently, however, a number of results have emerged in the theory of fuzzy sets, which are based on the application of fuzzy relations in suitable categories. A typical example of this use of fuzzy relations is the category $R_{L}($ Set $)$ of sets as objects and $\mathcal{L}$-valued fuzzy relations between sets as morphisms. This category is frequently used in approximation functors $R_{L}$ (Set) $\rightarrow C S L A T$, where CSLAT is the category of complete semilattices with semilattices homomorphisms. These functors then represent various approximations of fuzzy sets, defined by fuzzy relations. This approximation was for the first time defined by Goguen [2], when he introduced the notion of the image of a fuzzy set under a fuzzy relation. Many examples using explicitly or implicitly approximation functors can be found in rough fuzzy sets theory, F-transform theory and many others (see, e..g., [16, 17, 28, 29]).

In this paper we want to show what might be variants of categories with fuzzy objects, where morphisms are fuzzy relations. We use the idea of L.A.Manes [10], who defined the notion of a $\mathbf{T}$-relation from an object $\mathrm{X}$ to $\mathrm{Y}$ in a category $\mathbf{K}$ as a morphism in the Kleisli category $\mathbf{K}_{\mathbf{T}}$, where $\mathbf{T}$ is monad in $\mathbf{K}$. In that way we are able to define relations in many categories of fuzzy objects, including classical fuzzy sets, fuzzy sets in sets with similarity relations (i.e., in total fuzzy sets), or in spaces with fuzzy partitions as the ground structures for F-transforms and approximation functors.

\section{Preliminaries}

In this section we introduce principal notions and categories based on fuzzy sets which we use in the paper. A principal lattice structure used in fuzzy set theory in the paper is a complete residuated lattice (see e.g. [15]), i.e. a structure $\mathcal{L}=\left(L, \wedge, \vee, \otimes, \rightarrow, 0_{L}, 1_{L}\right)$ such that $(L, \wedge, \vee)$ is a complete lattice, $\left(L, \otimes, 1_{L}\right)$ is a commutative monoid with operation $\otimes$ isotone in both arguments and $\rightarrow$ is a binary operation which is residuated 
with respect to $\otimes$, i.e.

$$
\alpha \otimes \beta \leq \gamma \quad \text { iff } \quad \alpha \leq \beta \rightarrow \gamma
$$

If $\mathcal{L}$ is a complete residuated lattice, a $\mathcal{L}$-fuzzy set in a crisp set $X$ is a map $f: X \rightarrow L . f$ is a non-trivial $\mathcal{L}$-fuzzy set, if $f$ is not identical to the zero function. The core of a $\mathcal{L}$-fuzzy set $f$ in a set $X$ is defined by core $(f)=\left\{x \in X: f(x)=1_{L}\right\}$.

A set with $\mathcal{L}$-valued similarity relation (or $\mathcal{L}$-set) is a couple $(A, \delta)$, where $A$ is a set and $\delta: A \times A \rightarrow L$ is a map such that

(a) $(\forall x \in A) \quad \delta(x, x)=1$,

(b) $(\forall x, y \in A) \quad \delta(x, y)=\delta(y, x)$,

(c) $(\forall x, y, z \in A) \quad \delta(x, y) \otimes \delta(y, z) \leq \delta(x, z)$ (generalized transitivity).

In the paper we use some standard categories with (sometimes special) maps as morphisms. Namely,

1. Category CSLAT of complete $\vee$-semilattices as objects and with semilatices homomorphisms as morphisms.

2. Category Set of sets as objects with mappings as morphisms.

3. Category $\operatorname{Set}(\mathcal{L})$ with $\mathcal{L}$-sets $(X, \delta)$ as objects and with $f:(X, \delta) \rightarrow(Y, \gamma)$ as morphisms, where $f: X \rightarrow Y$ is a map and $\delta(x, y) \leq \gamma(f(x), f(y))$, $x, y \in X$.

Within morphisms in the category $\operatorname{Set}(\mathcal{L})$, we will be specially interested in morphisms $(A, \delta) \rightarrow(L, \leftrightarrow)$, which are called extensional sets and where $\leftrightarrow$ is the biresiduation operator in $\mathcal{L}$. Let $F(A, \delta)$ be the set of all such extensional fuzzy sets in $(A, \delta)$ in the category $\operatorname{Set}(\mathcal{L})$. We can define the covariant functor $F: \operatorname{Set}(\mathcal{L}) \rightarrow C S L A T(\vee)$, such that for any morphism $f:(A, \delta) \rightarrow(B, \gamma), f_{F}=F(f)$ is defined by

$s \in F(A, \delta), b \in B, \quad f_{F}(s)(b)=\bigvee_{x \in A} s(x) \otimes \gamma(f(x), b)$

Besides these well known categories we use also the category SpaceFP of spaces with fuzzy partitions which was introduce in [12] and which is the basic category for lattice-valued F-transforms. In the naxt part we recall the definition of the category SpaceFP, which is based on $\mathcal{L}$-valued fuzzy partitions, as it was introduced in [18] and some additional facts about objects and morphisms of that category.
Definition 2.1 ([18]) Let $X$ be a set. A system $\mathcal{A}=$ $\left\{A_{\lambda}: \lambda \in \Lambda\right\}$ of normal $\mathcal{L}$-valued fuzzy sets in $X$ is a fuzzy partition of $X$, if $\left\{\operatorname{core}\left(A_{\lambda}\right): \lambda \in \Lambda\right\}$ is a partition of $X$. A pair $(X, \mathcal{A})$ is called a space with a fuzzy partition. The index set of $\mathcal{A}$ will be denoted by $|\mathcal{A}|$.

Definition 2.2 The category SpaceFP is defined by

1. Fuzzy partitions $(X, \mathcal{A})$, as objects,

2. Morphisms $(g, \sigma):\left(X,\left\{A_{\lambda}: \lambda \in \Lambda\right\}\right) \rightarrow\left(Y,\left\{B_{\omega}:\right.\right.$ $\omega \in \Omega\})$, such that

(a) $g: X \rightarrow Y$ and $\sigma: \Lambda \rightarrow \Omega$ are maps,

(b) $\forall \lambda \in \Lambda, A_{\lambda}(x) \leq B_{\sigma(\lambda)}(g(x))$, for each $x \in$ $X$.

3. The composition of morphisms in $\mathbf{S p a c e F P}$ is defined by $(h, \tau) \circ(g, \sigma)=(h \circ g, \tau \circ \sigma)$.

In the paper [13] we proved the following proposition.

Proposition 2.1 There exists a full and faithful functor

$$
I: \operatorname{Set}(\mathcal{L}) \rightarrow \text { SpaceFP },
$$

which is also injective on objects.

We show how the object function of the functor $I$ is defined. Let $(X, \delta) \in \operatorname{Set}(\mathcal{L})$. Let the binary relation $\equiv{ }_{\delta}$ be defined by

$$
(\forall x, y \in X) \quad x \equiv_{\delta} y \leftrightarrow \delta(x, y)=1
$$

and let $X / \equiv_{\delta}$ be the partition of a set $X$ by the relation $\equiv_{\delta}$. We set

$$
\begin{gathered}
\mathcal{C}_{X, \delta}=\left\{C_{X, \mathbf{a}}: \mathbf{a} \in X / \equiv_{\delta}\right\}, \quad C_{X, \mathbf{a}}: X \rightarrow L, \\
(\forall x \in X) \quad C_{X, \mathbf{a}}(x)=\delta(x, y), \text { for any } y \in \mathbf{a} .
\end{gathered}
$$

Then $\left(X, \mathcal{C}_{X, \delta}\right) \in \mathbf{S p a c e F P}$ and the object function of the functor $I$ is defined by $I(X, \delta)=\left(X, \mathcal{C}_{X, \delta}\right)$.

For a bi-residuum operation $\leftrightarrow$ in $\mathcal{L}$, we have $(L, \leftrightarrow) \in$ $\operatorname{Set}(\mathcal{L})$ and we can put $(L, \mathcal{Q})=I(L, \leftrightarrow)$. It is then clear that $\mathcal{Q}=\left\{Q_{\alpha}: \alpha \in L\right\}$, where $Q_{\alpha}(\beta)=\alpha \leftrightarrow \beta$.

The following notion of an extensional fuzzy set in the category SpaceFP extends the notion of an extensional set in the category $\operatorname{Set}(\mathcal{L})$.

Definition 2.3 A map $f: X \rightarrow L$ is an extensional fuzzy set in a space with a fuzzy partition $(X, \mathcal{A})$ in the category SpaceFP, if there exists a map $\sigma:|\mathcal{A}| \rightarrow L$, such that $(f, \sigma)$ is a morphism $(X, \mathcal{A}) \rightarrow(L, \mathcal{Q})$ in the category SpaceFP. By $R(X, \mathcal{A})$ we denote the set of all extensional fuzzy sets in $(X, \mathcal{A})$. 
In the paper [13] we proved that if $f$ is an extensional fuzzy set in $(X, \mathcal{A})$, then there exists the unique map $[f]:|\mathcal{Q}| \rightarrow L$, such that $(f,[f])$ is a morphism in SpaceFP. The map $[f]$ is defined by

$$
\forall \lambda \in|\mathcal{A}|, \quad[f](\lambda)=f(x), \text { where } x \in \operatorname{core}\left(A_{\lambda}\right) .
$$

It can be proven that the definition of $[f]$ is correct, i.e., for arbitrary $x, x^{\prime} \in \operatorname{core}\left(A_{\lambda}\right), f(x)=f\left(x^{\prime}\right)$ holds (see [13]). Analogously, if $(f, \sigma):(X, \mathcal{A}) \rightarrow(L, \mathcal{Q})$ is a morphism in SpaceFP, then $f$ is uniquely determined by $\sigma$, i.e., $f=|\sigma|$, where

$\forall x \in X, \quad f(x)=|\sigma|(x)=\sigma(\lambda)$, where $x \in \operatorname{core}\left(A_{\lambda}\right)$.

In [13] we proved that for any space with a fuzzy partition $(X, \mathcal{A})$ it is possible to construct two $\mathcal{L}$-sets $\left(X, \delta_{X, \mathcal{A}}\right)$ and $\left(|\mathcal{A}|, \rho_{X, \mathcal{A}}\right)$, respectively, with similarity relations called characteristic similarity relations of $(X, \mathcal{A})$. The similarity relation $\delta_{X, \mathcal{A}}$ is the minimal similarity relation defined in $X$, such that

$$
\begin{gathered}
\forall x \in \operatorname{core}\left(A_{\lambda}\right), y \in \operatorname{core}\left(A_{\omega}\right), \\
\delta_{X, \mathcal{A}}(x, y) \geq \bigvee_{z \in \operatorname{core}\left(A_{\lambda}\right)} A_{\omega}(z) \vee \bigvee_{u \in \operatorname{core}\left(A_{\omega}\right)} A_{\lambda}(u),
\end{gathered}
$$

and

$$
\begin{gathered}
\forall \lambda, \alpha \in|\mathcal{A}|, \quad \rho_{X, \mathcal{A}}(\lambda, \alpha)=\delta_{X, \mathcal{A}}(x, y), \\
x \in \operatorname{core}\left(A_{\lambda}\right), y \in \operatorname{core}\left(A_{\alpha}\right) .
\end{gathered}
$$

Moreover, for these $\mathcal{L}$-sets we have

$$
\begin{gathered}
R(X, \mathcal{A})=F\left(X, \delta_{X, \mathcal{A}}\right) \\
\{[f]: f \in R(X, \mathcal{A})\}=F\left(|\mathcal{A}|, \rho_{X, \mathcal{A}}\right) .
\end{gathered}
$$

Hence, any extensional fuzzy set in $(X, \mathcal{A})$ is an extensional fuzzy set in $X$ with respect to the characteristic similarity relation $\delta_{X, \mathcal{A}}$.

\section{Categories with fuzzy relations as morphisms}

For each of the categories $\operatorname{Set}, \operatorname{Set}(\mathcal{L})$ and $\operatorname{SpaceFP}$ mentioned in the previous part, we can consider their modifications, where instead of maps as morphisms we will consider different variants of fuzzy relations.

The relational variant of a category $\mathbf{K}$ will be denoted by $R(\mathbf{K})$, except the category Set, where we have two variants of relational versions of this category. Namely, the category $R($ Set $)$ with classical relations as morphisms and the category $R_{L}($ Set $)$ with $\mathcal{L}$-valued fuzzy relations as morphisms.

Definition 3.1 The category $R($ Set $)$ is defined by
1. Objects are identical to objects of Set,

2. Morphisms $R: X \rightarrow Y$ are classical relations from $X$ to $Y$,

3. Composition of morphisms is a standard composition of relations.

Definition 3.2 The category $R_{L}($ Set $)$ is defined by

1. Objects are identical to objects of Set.

2. Morphisms $R: X \rightarrow Y$ are $\mathcal{L}$-valued fuzzy relations $R \subsetneq X \times Y$,

3. A composition of morphisms $R: X \rightarrow Y, S$ : $Y \rightarrow Z$ is defined by

$\forall x \in X, z \in Z, \quad S \circ R(x, z)=\bigvee_{y \in Y} R(x, y) \otimes S(y, z)$

Definition 3.3 The category $R(\operatorname{Set}(\mathcal{L}))$ is defined by

1. Objects are identical to objects of $\operatorname{Set}(\mathcal{L})$,

2. Morphisms $R:(X, \delta) \rightarrow(Y, \gamma)$ are $\mathcal{L}$-valued extensional fuzzy relation $R \cong X \times Y$, i.e.,

$$
\begin{aligned}
& R(x, y) \otimes \delta\left(x, x^{\prime}\right) \leq R\left(x^{\prime}, y\right), \\
& R(x, y) \otimes \gamma\left(y, y^{\prime}\right) \leq R\left(x, y^{\prime}\right),
\end{aligned}
$$

for arbitrary $x, x^{\prime} \in X, y, y^{\prime} \in Y$.

3. Composition of morphisms is the same as in the category $R(\operatorname{Set}(\mathcal{L}))$.

To define the relational version of the category SpaceFP we need to use the characteristic similarity relations $\delta_{X, \mathcal{A}}$ and $\rho_{X, \mathcal{A}}$ of spaces with fuzzy partitions $(X, \mathcal{A})$.

Definition 3.4 The category $R(\mathbf{S p a c e F P})$ is defined by

1. Objects are identical to objects of SpaceFP,

2. Morphisms $(R, S):(X, \mathcal{A}) \rightarrow(Y, \mathcal{B})$, where $\mathcal{A}=$ $\left\{A_{i}: i \in|\mathcal{A}|\right\}, \mathcal{B}=\left\{B_{j}: j \in|\mathcal{B}|\right\}$, are defined by

(a) $R$ and $S$ are $\mathcal{L}$-valued fuzzy relarions $R \cong$ $X \times Y, S \cong|\mathcal{A}| \times|\mathcal{B}|$

(b) For each $x \in X, j, j^{\prime} \in|\mathcal{B}|, y, y^{\prime} \in Y$ hold

$$
\begin{gathered}
A_{i}(x) \leq R(x, y) \leftrightarrow S\left(i, j_{y}\right), \\
R(x, y) \otimes \delta_{Y, \mathcal{B}}\left(y, y^{\prime}\right) \leq R\left(x, y^{\prime}\right), \\
S(i, j) \otimes \rho_{Y, \mathcal{B}}\left(j, j^{\prime}\right) \leq S\left(i, j^{\prime}\right),
\end{gathered}
$$

where $j_{y} \in|\mathcal{B}|$ is such that $y \in \operatorname{core}\left(B_{j_{y}}\right)$. 
3. Composition of morphisms $(R, S):(X, \mathcal{A}) \rightarrow$ $(Y, \mathcal{B})$ and $\left(R^{\prime}, S^{\prime}\right):(Y, \mathcal{B}) \rightarrow(Z, \mathcal{C})$ is defined by

$$
\left(R^{\prime}, S^{\prime}\right) \cdot(R, S)=\left(R^{\prime} \circ R, S^{\prime} \circ S\right),
$$

where $\circ$ is the composition of relations from $R_{L}($ Set $)$.

Obviously, categories $R(\mathbf{K})$ retain some features of the original categories and relationships between the different categories. An example of preserving these relationships may be the transfer of embedding functors between categories

$$
\text { Set } \hookrightarrow \operatorname{Set}(\mathcal{L}) \hookrightarrow \text { SpaceFP. }
$$

to embedding functors between the relational versions of these categories

In fact, the following simple lemma holds.

Lemma 3.1 There exist the following embedding functors

$$
R(\text { Set }) \hookrightarrow R_{L}(\text { Set }) \hookrightarrow R(\operatorname{Set}(\mathcal{L})) \hookrightarrow R(\text { SpaceFP }) .
$$

Whatever the Definitions 3.1 - Definition 3.4 of the categories with relations as morphisms seem to be natural, it is possible to ask why these definitions are chosen and not the other. In order to answer this question, we should set out clear rules on what can be such a definition of a relation or a fuzzy relation in a category.

An we mentioned in the Introduction, one of the possibilities how to define relations as morphisms in a category is to use the approach introduced by Manes in the published review [10]. In this concept of relations in a category $\mathbf{K}$, a relation is not defined absolutely, it is tied to the existence of a monad in this category. Therefore to different monads $\mathbf{T}$ in a category $\mathbf{K}$, different types of relations between objects in this category, called $\mathbf{T}$-relations, can be defined in $\mathbf{K}$. This approach allows to link the theory of categories with relations as morphisms with more general structures, i.e., monads in categories, while at the same time allowing to justify the more general nature of the choice of the specific type of relations in the category representing morphisms.

For the convenience of readers we firstly repeat some basic definitions of monads and corresponding Kleisli categories, which are necessary for the definition of relations in a category. The idea of using monads for fuzzyfication is based on extension of objects $X$ of a category $\mathbf{K}$ to another object $T(X) \in \mathbf{K}$, which may be regarded as a "cloud of fuzzy states" with a morphism $\eta: X \rightarrow T(X)$, representing "crisp" states in the object of "fuzzy states". Then a "fuzzy morphism" $f: X \rightsquigarrow Y$ is simply a morphism $f: X \rightarrow T(Y)$ in the category $\mathbf{K}$ and a composition of fuzzy morphisms is defined by a special operation $\diamond$. The result of these constructions is a triple $\mathbf{T}=(T, \diamond, \eta)$, which Manes [9] called a fuzzy theory and which, in fact, is a monad (or algebraic theory) in a clone form.

We present the definitions of all these notions.

Definition 3.5 (E.G.Manes [9]) $\mathbf{T}=(T, \diamond, \eta)$ is a monad (in clone form) (or algebraic theory (in clone form)) in a category $\mathbf{K}$, if

(1) $T: \mathbf{K} \rightarrow \mathbf{K}$ is an object function,

(2) $\eta$ is a system of $\mathbf{K}$-morphisms $\eta_{A}: A \rightarrow T(A)$, for any object $A$,

(3) for each pair of K-morphisms $f: A \rightarrow T(B)$, $g: B \rightarrow T(C)$, there exists a composition (called a Kleisli composition) $g \diamond f: A \rightarrow T(C)$, which is associative,

(4) for every $\mathbf{K}$-morphism $f: A \rightarrow T(B), \eta_{B} \diamond f=f$,

$(5) \diamond$ is compatible with composition of morphisms of $\mathbf{K}$, i.e., for all $\mathbf{K}$-morphisms $f: A \rightarrow B, g: B \rightarrow$ $T(C)$, we have $g \diamond\left(\eta_{B} \cdot f\right)=g . f$.

For a monad $\mathbf{T}$ in a category $\mathbf{K}$ a new category $\mathbf{K}_{\mathbf{T}}$ can be defined, called the Kleisli category. We recall a definition of $\mathbf{K}_{\mathbf{T}}$ (see, e.g., [8],[9]).

Definition 3.6 ([9]) Let $\mathbf{K}$ be a category and let $\mathbf{T}=$ $(T, \diamond, \eta)$ be a monad (in clone form) in $\mathbf{K}$. Then the Kleisli category $\mathbf{K}_{\mathbf{T}}$ of $\mathbf{T}$ is defined by

1. Objects of $\mathbf{K}_{\mathbf{T}}=$ objects of $\mathbf{K}$,

2. For any objects $A, B \in \mathbf{K}, H_{0} m_{\mathbf{K}_{\mathbf{T}}}(A, B)=$ Hom $_{\mathbf{K}}(A, T(B))$. Morphisms in $\mathbf{K}_{\mathbf{T}}$ are denoted by $A \rightsquigarrow B$.

3. A composition of morphisms $f: A \rightsquigarrow B, g: B \rightsquigarrow$ $C$ is defined by $g \diamond f$.

It should be noted that for any morphism $R: X \rightsquigarrow Y$ in $\mathbf{K}_{\mathbf{T}}, R$ is identical to the morphism $R^{*}: X \rightarrow$ $T(Y)$ in the category $\mathbf{K}$ and there exists the morphism $R^{\#}: T(X) \rightarrow T(Y)$ in the category $\mathbf{K}$, defined by $R^{\#}=R^{*} \diamond 1_{T(X)}$.

Now, according to Manes, we can define T-relations in a category.

Definition 3.7 Let $\mathbf{K}$ be a category and let $\mathbf{T}$ be a monad in $\mathbf{K}$. A T $\mathbf{T}$-relation $R$ from an object $X$ to an object $Y$ in $\mathbf{K}$ is a morphism $R: X \rightsquigarrow Y$ in the Kleisli category $\mathbf{K}_{\mathbf{T}}$, or, equivalently, the morphism $R^{*}: X \rightarrow T(X)$ in the category $\mathbf{K}$. 
From the above definition it follows that the Kleisli category $\mathbf{K}_{\mathbf{T}}$ can be considered the relational variant of a category $\mathbf{K}$ where instead of morphisms of category $\mathrm{K}, \mathbf{T}$-relations are used. Hence, instead of $\mathbf{K}_{\mathbf{T}}$, we can also use the more intuitive equivalent notation $R_{\mathbf{T}}(\mathbf{K})$.

To justify the definitions of relations presented in Definition 3.1 - Definition 3.4 we show that all these relations are, in fact, $\mathbf{T}$-relations for appropriate monad in the corresponding category.

Theorem 3.1 Let $\mathbf{K}$ be any of categories $\operatorname{Set}, \operatorname{Set}(\mathcal{L})$ or $\mathbf{S p a c e F P . ~ T h e n ~ f o r ~ a n y ~ r e l a t i o n a l ~ v a r i a n t ~} \mathbf{L}$ of the category $\mathbf{K}$ from the Definition 3.1 - Definition 3.4 there exists a monad $\mathbf{T}$ in the category $\mathbf{K}$ such that

$$
\mathbf{L} \cong \mathbf{K}_{\mathbf{T}}
$$

Proof. Monads $\mathbf{T}$ for individual categories $\mathbf{K}$ mentioned in Theorem were introduced in various papers. Recall $[9,21,24]$ for monads $\mathbf{P}$ and $\mathbf{Z}$ in the category Set, [11] for the monad $\mathbf{F}$ in the category $\operatorname{Set}(\mathcal{L})$ and [14] for the monad $\mathbf{R}$ in the category SpaceFP. To unify the notations we repeat the basic definitions of these monads.

(Part I) The monad $\mathbf{P}=(P, \diamond, \eta)$ in the category Set is defined by

(1) For each object $X \in \mathbf{S e t}, P(X)=2^{X}$,

(2) For each objects $X \in$ Set, $\eta_{X}: X \rightarrow P(X)$ by $\eta_{X}(x)=\{x\}$.

(3) For each $f: X \rightarrow P(Y), g: Y \rightarrow P(Z), g \diamond f:$ $X \rightarrow P(Z)$, by

$$
(g \diamond f)(x)=\bigcup_{y \in f(x)} g(y) .
$$

Then, we have

$$
R(\text { Set }) \cong \operatorname{Set}_{\mathbf{P}}
$$

In fact, let $R: X \rightarrow Y$ be a morphism in $R$ (Set), the functor $J_{\mathbf{P}}: R($ Set $) \rightarrow$ Set $_{\mathbf{P}}$ is defined by

$$
\begin{gathered}
J_{\mathbf{P}}(X)=X, \quad J_{\mathbf{P}}(R): X \rightsquigarrow Y, \\
x \in X, \quad J_{\mathbf{P}}(R)(x)=\{y \in Y:(x, y) \in R\} \in P(X) .
\end{gathered}
$$

It can be proven easy that $J_{\mathbf{P}}$ is the isomorphic functor.

(Part II) The monad $\mathbf{Z}=(Z, \diamond, \chi)$ in the category Set is defined by

(1) For each object $X \in$ Set, the object function $Z$ : Set $\rightarrow$ Set is defined by $Z(X)=L^{X}$,
(2) For each object $X \in$ Set, $\chi_{X}: X \rightarrow L^{X}$ is a characteristic map $\chi_{X,\{x\}}: X \rightarrow L$ of $\{x\}$ in $X$,

(3) For each $f: X \rightarrow L^{Y}$ and $g: Y \rightarrow L^{Z}$ in Set, $g \diamond f: X \rightarrow L^{Z}$ is defined by

$$
[(g \diamond f)(x)](z)=\bigvee_{y \in Y}(f(x))(y) \otimes(g(y))(z) .
$$

Then, we have

$$
R_{L}(\text { Set }) \cong \operatorname{Set}_{\mathbf{Z}}
$$

In fact, let $R: X \rightarrow Y$ be a morphism in $R_{L}$ (Set), the functor $J_{\mathbf{Z}}: R_{L}(\mathbf{S e t}) \rightarrow \mathbf{S e t}_{\mathbf{Z}}$ is defined by

$$
\begin{aligned}
& J_{\mathbf{Z}}(X)=X, \quad J_{\mathbf{Z}}(R): X \rightsquigarrow Y \text { in } \operatorname{Set}_{\mathbf{Z}}, \\
& x \in X, y \in Y, \quad J_{\mathbf{Z}}(R)(x)(y)=R(x, y) .
\end{aligned}
$$

Then $J_{\mathbf{Z}}$ is a functor, as follows from a simple verification of the equalities

$$
J_{\mathbf{Z}}(S \circ R)=J_{\mathbf{Z}}(S) \diamond J_{\mathbf{Z}}(R), \quad J_{\mathbf{Z}}\left(1_{X}\right)=\chi_{X},
$$

for arbitrary morphisms $R: X \rightarrow Y, S: Y \rightarrow Z$ in $R_{L}$ (Set) and the diagonal relation $1_{X}: X \rightarrow X$ as the identity morphism. It is clear that $J_{\mathbf{Z}}$ is also the isomorphic functor.

(Part III) The monad $\mathbf{F}=(\mathcal{F}, \diamond, \eta)$ in the category $\operatorname{Set}(\mathcal{L})$ is defined by

(1) The object function $\mathcal{F}: \operatorname{Set}(\mathcal{L}) \rightarrow \operatorname{Set}(\mathcal{L})$ is defined by $\mathcal{F}(X, \delta)=\left(F(X, \delta), \sigma_{X, \delta}\right)$, where the similarity relation $\sigma$ is defined by $\sigma_{X, \delta}(s, t)=$ $\bigwedge_{x \in X} s(x) \leftrightarrow t(x)$, for all $s, t \in F(X, \delta)$,

(2) For each $(X, \delta) \in \operatorname{Set}(\mathcal{L}), \eta_{(X, \delta)}:(X, \delta) \rightarrow$ $\mathcal{F}(X, \delta)$ is defined by $\eta_{(X, \delta)}(a)(x)=\delta(a, x)$, for all $a, x \in X$,

(3) For each $f:(X, \delta) \rightarrow \mathcal{F}(Y, \gamma)$, for each $g$ : $(Y, \gamma) \rightarrow \mathcal{F}(Z, \omega)$ in $\operatorname{Set}(\mathcal{L})$, define $g \diamond f:$ $(X, \delta) \rightarrow \mathcal{F}(Z, \omega)$ by

$$
[(g \diamond f)(x)](z)=\bigvee_{y \in Y}(f(x))(y) \otimes(g(y))(z) .
$$

Then, we have

$$
R(\operatorname{Set}(\mathcal{L})) \cong \operatorname{Set}(\mathcal{L})_{\mathbf{F}}
$$

In fact, let $R:(X, \delta) \rightarrow(Y, \gamma)$ be a morphism in $R(\operatorname{Set}(\mathcal{L}))$. The functor $J_{\mathbf{F}}: R(\operatorname{Set}(\mathcal{L})) \rightarrow \operatorname{Set}(\mathcal{L})_{\mathbf{F}}$ is defined by

$$
\begin{gathered}
J_{\mathbf{F}}(X, \delta)=(X, \delta) \quad J_{\mathbf{F}}(R):(X, \delta) \rightsquigarrow(Y, \gamma) \text { in } \operatorname{Set}(\mathcal{L})_{\mathbf{F}}, \\
J_{\mathbf{F}}(R):(X, \delta) \rightarrow\left(F(Y, \gamma), \sigma_{Y, \gamma}\right), \operatorname{in} \operatorname{Set}(\mathcal{L}), \\
x \in(X, \delta), y \in(Y, \gamma), \quad J_{\mathbf{F}}(R)(x)(y):=R(x, y) .
\end{gathered}
$$


From the properties of the morphism $R$ in $\operatorname{Set}(\mathcal{L})$ it follows that $J_{\mathbf{F}}(R)(x) \in F(Y, \gamma)$, for each $x \in$ $X$. Moreover, $J_{\mathbf{F}}(R)$ is a morphism in the category $\operatorname{Set}(\mathcal{L})$. In fact, we have

$$
\begin{gathered}
\sigma_{Y, \gamma}\left(J_{\mathbf{F}}(R)(x), J_{\mathbf{F}}(R)\left(x^{\prime}\right)\right)= \\
\bigwedge_{y \in Y} J_{\mathbf{F}}(R)(x)(y) \leftrightarrow J_{\mathbf{F}}(R)\left(x^{\prime}\right)(y)= \\
\bigwedge_{y \in Y} R(x, y) \leftrightarrow R\left(x^{\prime}, y\right) \leq \delta\left(x, x^{\prime}\right) .
\end{gathered}
$$

We show that $J_{\mathbf{F}}$ is a functor. In fact, let $R:(X, \delta) \rightarrow$ $(Y, \gamma)$ and $S:(Y, \gamma) \rightarrow(Z, \tau)$ be morphisms in $\operatorname{Set}(\mathcal{L})$. As in the case (II),

$$
J_{\mathbf{F}}(S \circ R)=J_{\mathbf{F}}(S) \diamond J_{\mathbf{F}}(R)
$$

holds. The identity morphism $1_{(X, \delta)}$ in the category $\operatorname{Set}(\mathcal{L})$ is defined by

$$
x, y \in X, \quad 1_{(X, \delta)}(x, y)=\delta(x, y),
$$

and we have $J_{\mathbf{F}}\left(1_{(X, \delta)}\right)=\eta_{(X, \delta)}=1_{\mathcal{F}(X, \delta)}$ in the category $\operatorname{Set}(\mathcal{L})_{\mathbf{F}}$. To show that $J_{\mathbf{F}}$ is the isomorphic functor, let $f:(X, \delta) \rightsquigarrow(Y, \gamma)$ be a morphism in the category $\operatorname{Set}(\mathcal{L})_{\mathbf{F}}$, i.e., $f:(X, \delta) \rightarrow\left(F(Y, \gamma), \sigma_{Y, \gamma}\right)$ be a morphism in $\operatorname{Set}(\mathcal{L})$. Let $R$ be defined by $R(x, y)=f(x)(y)$, for arbitrary $x \in X, y \in Y$. Then, since $f$ is a morphism, $R:(X, \delta) \rightarrow(Y, \gamma)$ is a morphism in the category $\operatorname{Set}(\mathcal{L})$ and $J_{\mathbf{F}}(R)=f$.

(Part IV) Let $(X, \mathcal{A})$ be a set with a fuzzy partition. According to the Definition 2.3 and relation (1), we have $R(X, \mathcal{A})=F\left(X, \delta_{X, \mathcal{A}}\right)$. On $R(X, \mathcal{A})$ we define the fuzzy relation $\theta_{X, \mathcal{A}}$ by

$$
\theta_{X, \mathcal{A}}(s, t)=\bigwedge_{x \in X} s(x) \leftrightarrow t(x),
$$

for each $s, t \in R(X, \mathcal{A})$. Then $\theta_{X, \mathcal{A}}$ is a similarity relation on $R(X, \mathcal{A})$ and, according to Proposition 2.1, we can consider the space with a fuzzy partition $\mathcal{R}(X, \mathcal{A})=\left(R(X, \mathcal{A}), \mathcal{C}_{\left.R(X, \mathcal{A}), \theta_{X, \mathcal{A}}\right)=}\right.$ $I\left(R(X, \mathcal{A}), \theta_{X, \mathcal{A}}\right) . \quad$ It is clear that $\equiv_{\theta_{X, \mathcal{A}}}$ is the

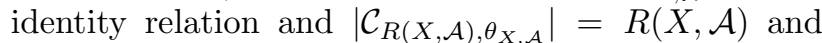
$\mathcal{C}_{R(X, \mathcal{A}), \theta_{X, \mathcal{A}}}=\left\{C_{f}: f \in R(X, \mathcal{A})\right\}$, where $C_{f}(g)=$ $\theta_{X, \mathcal{A}}(f, g)$. For simplicity, instead of $\mathcal{C}_{R(X, \mathcal{A}), \theta_{X, \mathcal{A}}}$ we use only $\mathcal{C}_{X, \mathcal{A}}$. For the characteristic similarity relation of a set with a fuzzy partition $\mathcal{R}(X, \mathcal{A})$ we can prove

$$
\delta_{\mathcal{R}(X, \mathcal{A})}=\theta_{X, \mathcal{A}}
$$

Then the monad $\mathbf{R}=(\mathcal{R}, \square, \nu)$ is defined by

(1) Object function $\mathcal{R}:$ SpaceF $\rightarrow$ SpaceFP, defined by

$$
\mathcal{R}(X, \mathcal{A})=I\left(R(X, \mathcal{A}), \theta_{X, \mathcal{A}}\right)=\left(R(X, \mathcal{A}), \mathcal{C}_{X, \mathcal{A}}\right),
$$

(2) For each object $(X, \mathcal{A}) \in$ SpaceFP,$\nu_{X, \mathcal{A}}=$ $(e, \varepsilon):(X, \mathcal{A}) \rightarrow \mathcal{R}(X, \mathcal{A})$ is a morphism in SpaceFP, such that

$$
e: X \rightarrow R(X, \mathcal{A}), \quad \varepsilon:|\mathcal{A}| \rightarrow\left|\mathcal{C}_{X, \mathcal{A}}\right|=R(X, \mathcal{A}),
$$

defined by

$$
\begin{gathered}
\forall x, z \in X, \quad e(x)(z)=\delta_{X, \mathcal{A}}(x, z), \\
\forall \lambda \in|\mathcal{A}|, z \in X, \quad \varepsilon(\lambda)(z)=\rho_{X, \mathcal{A}}(\lambda, \alpha), \\
\text { if } z \in \operatorname{core}\left(A_{\alpha}\right),
\end{gathered}
$$

(3) For each morphisms $(f, u):(X, \mathcal{A}) \rightarrow \mathcal{R}(Y, \mathcal{B})$ and $(g, v):(Y, \mathcal{B}) \rightarrow \mathcal{R}(Z, \mathcal{D})$ in SpaceFP, define $(g, v) \square(f, u):(X, \mathcal{A}) \rightarrow \mathcal{R}(Z, \mathcal{D})$ by

$$
\begin{gathered}
(g, v) \square(f, u)=(g \Delta f, v \nabla u), \\
\forall x \in X, z \in Z, \quad[(g \Delta f)(x)](z)= \\
\bigvee_{y \in Y}(f(x))(y) \otimes(g(y))(z), \\
\forall \lambda \in \Lambda, z \in Z, \quad[(v \nabla u)(\lambda)](z)= \\
\bigvee_{y \in Y} u(\lambda)(y) \otimes v\left(\omega_{y}\right)(z),
\end{gathered}
$$

where $\omega_{y}$ is the unique index in $\mathcal{B}$, such that $y \in$ $\operatorname{core}\left(B_{\omega_{y}}\right)$.

According to [14], $\mathbf{R}$ is a monad in the category SpaceFP and we have

$$
R(\mathbf{S p a c e F P}) \cong \mathbf{S p a c e F P}_{\mathbf{R}}
$$

In fact, let $(T, S):(X, \mathcal{A}) \rightarrow(Y, \mathcal{B})$ be a morphism in $R($ SpaceFP $)$. The functor $J_{\mathbf{R}}: R($ SpaceFP $) \rightarrow$ SpaceFP $_{\mathbf{R}}$ is defined by

$$
\begin{gathered}
J_{\mathbf{R}}(X, \mathcal{A})=(X, \mathcal{A}) \quad J_{\mathbf{R}}(T, S):(X, \mathcal{A}) \rightsquigarrow(Y, \mathcal{B}) \\
\quad \text { in } \mathbf{S p a c e F P}_{\mathbf{R}}, \text { i.e., } \\
J_{\mathbf{R}}(T, S)=(f, \tau):(X, \mathcal{A}) \rightarrow\left(R(Y, \mathcal{B}), \mathcal{C}_{Y, \mathcal{B}}\right), \\
\text { in SpaceFP, } \\
f: X \rightarrow R(Y, \mathcal{B}), \quad \tau:|\mathcal{A}| \rightarrow\left|\mathcal{C}_{Y, \mathcal{B}}\right|=R(Y, \mathcal{B}), \\
x \in X, y \in Y, i \in|\mathcal{A}|, \quad f(x)(y):=T(x, y), \\
\tau(i)(y):=S\left(i, j_{y}\right),
\end{gathered}
$$

where $j_{y} \in|\mathcal{B}|$ is such that $y \in \operatorname{core}\left(B_{j_{y}}\right)$. Let $\mathcal{A}=$ $\left\{A_{i}: i \in|\mathcal{A}|\right\}$ and $\mathcal{B}=\left\{B_{j}: j \in|\mathcal{B}|\right\}$. From the definition of relations in SpaceFP it follows that for arbitrary $x \in X, y \in Y, i \in|\mathcal{A}|, j \in|\mathcal{B}|$, we have

$$
\begin{gathered}
f(x), \tau(i) \in R(Y, \mathcal{B}), \\
A_{i}(x) \leq T(x, y) \leftrightarrow S\left(i, j_{y}\right)=f(x)(y) \leftrightarrow \tau(i)(y) .
\end{gathered}
$$

Then, for arbitrary $x \in X, i \in|\mathcal{A}|$, we have

$$
\begin{gathered}
C_{\tau(i)}(f(x))=\theta_{Y, \mathcal{B}}(\tau(i), f(x))= \\
\bigwedge_{y \in Y} \tau(i)(y) \leftrightarrow f(x)(y) \geq A_{i}(x)
\end{gathered}
$$


and $J_{\mathbf{R}}(T, S)$ is a morphism in SpaceFP. Moreover, it can be proved that

$$
\begin{gathered}
J_{\mathbf{R}}\left(T^{\prime} \circ T, S^{\prime} \circ S\right)=J_{\mathbf{R}}\left(\left(T^{\prime}, S^{\prime}\right) \circ(T, S)\right)= \\
J_{\mathbf{R}}\left(T^{\prime}, S^{\prime}\right) \square J_{\mathbf{R}}(T, S) .
\end{gathered}
$$

and $J_{\mathbf{R}}\left(1_{(X, \mathcal{A})}\right)=\nu_{(X, \mathcal{A})}$. Hence $J_{\mathbf{R}}$ is a functor.

The inverse functor $J_{\mathbf{R}}^{-}: \mathbf{S p a c e F} \mathbf{P}_{\mathbf{R}} \rightarrow R(\mathbf{S p a c e F P})$ can be defined similarly. Let $(f, \tau):(X, \mathcal{A}) \rightsquigarrow(Y, \mathcal{B})$ be a morphism in the category $\mathbf{S p a c e F P}_{\mathbf{R}}$, i.e., the morphism $(X, \mathcal{A}) \rightarrow\left(R(Y, \mathcal{B}), \mathcal{C}_{Y, \mathcal{B}}\right)$ in SpaceFP. Then we set

$$
\begin{gathered}
x \in X, y \in Y, i \in|\mathcal{A}|, j \in|\mathcal{B}|, \quad T(x, y):=f(x)(y), \\
S(i, j):=\tau(i)\left(y_{j}\right),
\end{gathered}
$$

where $y_{j} \in \operatorname{core}\left(B_{j}\right)$. We show that $(T, S):(X, \mathcal{A}) \rightarrow$ $(Y, \mathcal{B})$ is a morphism in $R(\mathbf{S p a c e F P})$, Firstly, the following relation holds:

$$
x, x^{\prime} \in X, \quad \delta_{X, \mathcal{A}}\left(x, x^{\prime}\right) \leq \theta_{Y, \mathcal{B}}\left(f(x), f\left(x^{\prime}\right)\right) .
$$

Let $x \in \operatorname{core}\left(A_{i_{x}}\right), x^{\prime} \in \operatorname{core}\left(A_{i_{x^{\prime}}}\right)$. Since $(f, \tau)$ is a morphism in SpaceFP, we have

$$
1_{L}=A_{i_{x}}(x) \leq C_{\tau\left(i_{x}\right)}(f(x)),
$$

and it follows that $f(x) \in f\left(\operatorname{core}\left(A_{i_{x}}\right)\right) \subseteq$ core $\left(C_{\tau}\left(i_{x}\right)\right)$. Analogously, we obtain $f\left(x^{\prime}\right) \in$ $\operatorname{core}\left(C_{\tau\left(i_{x^{\prime}}\right)}\right)$. Then, according to the definition of characteristic similarity relation in spaces with fuzzy partitions, we have

$$
\begin{aligned}
& \delta_{\mathcal{R}(X, \mathcal{A})}\left(f(x), f\left(x^{\prime}\right)\right) \geq \\
& \bigvee_{r \in \operatorname{core}\left(C_{\tau\left(i_{x}\right)}\right)} C_{\tau\left(i_{x^{\prime}}\right)}(r) \vee \underset{t \in \operatorname{core}\left(C_{\tau\left(i_{x^{\prime}}\right)}\right)}{ } C_{\tau\left(i_{x}\right)}(t) \geq \\
& \bigvee_{b \in \operatorname{core}\left(A_{i_{x}}\right)} C_{\tau\left(i_{x^{\prime}}\right)}(f(b)) \vee \bigvee_{b^{\prime} \in \operatorname{core}\left(A_{i_{x^{\prime}}}\right)} C_{\tau\left(i_{x}\right)}\left(f\left(b^{\prime}\right)\right) \geq \\
& \bigvee_{b \in \operatorname{core}\left(A_{i_{x}}\right)} A_{i_{x^{\prime}}}(b) \vee \bigvee_{b^{\prime} \in \operatorname{core}\left(A_{i_{x^{\prime}}}\right)} A_{i_{x}}\left(b^{\prime}\right) .
\end{aligned}
$$

Then, from the definition of $\delta_{X, \mathcal{A}}$, it follows that $\delta_{\mathcal{R}(Y, \mathcal{B})}\left(f(x), f\left(x^{\prime}\right)\right) \geq \delta_{X, \mathcal{A}}\left(x, x^{\prime}\right)$. Hence, we obtain

$\delta_{X, \mathcal{A}}\left(x, x^{\prime}\right) \leq \delta_{\mathcal{R}(Y, \mathcal{B})}\left(f(x), f\left(x^{\prime}\right)\right)=\theta_{Y, \mathcal{B}}\left(f(x), f\left(x^{\prime}\right)\right)$,

and the relation (3) holds.

Now, since $f(x) \in R(Y, \mathcal{B})$ for arbitrary $x \in X, T$ is extensional with respect to $\delta_{Y, \mathcal{B}}$. Let $i \in|\mathcal{A}|, j, j^{\prime} \in$ $|\mathcal{B}|$. Then, we have

$$
\begin{gathered}
S(i, j) \otimes \rho_{Y, \mathcal{B}}\left(j, j^{\prime}\right)=\tau(i)\left(y_{j}\right) \otimes \delta_{Y, \mathcal{B}}\left(y_{j}, y_{j^{\prime}}\right) \leq \\
\tau(i)\left(y_{j^{\prime}}\right)=S\left(i, j^{\prime}\right),
\end{gathered}
$$

as follows from the relation (1). Further, since $(f, \tau)$ is a morphism, we obtain

$$
\begin{gathered}
A_{i}(x) \leq C_{\tau(i)}(f(x)) \leq f(x)(y) \leftrightarrow \tau(i)(y)= \\
T(x, y) \leftrightarrow S\left(i, y_{j}\right),
\end{gathered}
$$

and $(T, S)$ is a relation in SpaceFP. Let $(f, \tau)$ : $(X, \mathcal{A}) \rightsquigarrow(Y, \mathcal{B})$ and $\left(f^{\prime}, \tau^{\prime}\right):(Y, \mathcal{B}) \rightsquigarrow(Z, \mathcal{D})$ be morphisms in $\mathbf{S p a c e F P}_{\mathbf{R}}$,

$$
\left(f^{\prime}, \tau^{\prime}\right) \square(f, \tau)=\left(f^{\prime} \triangle f, \tau^{\prime} \nabla \tau\right):(X, \mathcal{A}) \rightsquigarrow(Z, \mathcal{D}) .
$$

Then we have

$$
J_{\mathbf{R}}^{-}\left(f^{\prime} \triangle f, \tau^{\prime} \nabla \tau\right)=J_{\mathbf{R}}^{-}\left(f^{\prime}, \tau^{\prime}\right) \circ J_{\mathbf{R}}^{-}(f, \tau),
$$

as it can be verified by a simple calculation, analogously to the functor $J_{\mathbf{R}}$. Finally, since $J_{\mathbf{R}}^{-}\left(\nu_{(X, \mathcal{A})}\right)=$ $J_{\mathbf{R}}^{-}(e, \varepsilon)=\left(\delta_{X, \mathcal{A}}, \rho_{X, \mathcal{A}}\right)=1_{(X, \mathcal{A})}$ in the category $R(\mathbf{S p a c e F P})$. Hence, $J_{\mathbf{R}}^{-}$is a functor and the inverse functor to $J_{\mathbf{R}}$.

Corollary 3.1 For arbitrary category $\mathbf{K}$ from Definitions 3.1-3.4 there exists embedding functors $\mathbf{K} \hookrightarrow$ $R(\mathbf{K})$.

Proof. Let $\mathbf{T}$ be the monad such that $\mathbf{K}_{\mathbf{T}} \cong R(\mathbf{K})$. There exists an embedding functor $I_{\mathbf{T}}: \mathbf{K} \hookrightarrow \mathbf{K}_{\mathbf{T}}$, such that

$$
I_{\mathbf{K}}(X)=X, \quad I_{\mathbf{T}}(f: X \rightarrow Y)=\eta_{Y} \cdot f: X \rightsquigarrow Y .
$$

Hence, $\mathbf{K} \hookrightarrow \mathbf{K}_{\mathbf{T}} \cong R(\mathbf{K})$.

\section{Acknowledgement}

This research was partially supported from the project GA18-06915S provided by the Grant Agency of the Czech Republic and from the ERDF/ESF project CZ.02.1.01/0.0/0.0/17-049/0008414.

\section{References}

[1] Barr, M., Fuzzy sets and topos theory, Canad. Math. Bull. 29(4)(1986), 501-508.

[2] Goguen, J.A., L-fuzzy sets, J. Math. Anal. Appl. 18(1967), 145-174.

[3] Harding, J., Walker, C., Walker, E., Categories with fuzzy sets and relations, Fuzzy Sets and Systems 256()2014), 149-165.

[4] Höhle, U., M-Valued sets and sheaves over integral commutative cl-monoids, S. E. Rodabaugh et al. (eds.), Applications of Category Theory to Fuzzy Subsets , 33-72, Kluwer Academic Publishers, 1992. 
[5] Höhle, U., GL-Quantales: Q-Valued Sets and Their Singletons, Studia Logica 61(1998), 123148.

[6] Höhle, U., Kubiak, T., Quantale Sets and Their Singleton Monad, Preprint, 2008.

[7] Höhle, U., Stout, L. N., Foundations of Fuzzy Sets, Fuzzy Sets and Systems 40(1991), 257-296.

[8] Kleisli, H., Every standard construction is induced by a pair of adjoint functors. Proc. American Math. Soc. 16(1965), 544-546.

[9] Manes, E.G., Algebraic Theories, Springer Verlag, Berlin, 1976.

[10] Manes, L.A., Book review Fuzzy sets and systems, Theory and applications, Bulletin (New Series) of the American Mathematica Society 7(3)(1982).

[11] Močkoř, J., Powerset Operators of Extensional Fuzzy sets, Iranian Journal of Fuzzy Systems 15(2)(2018), 143-163.

[12] Močkoř, J., Spaces with fuzzy partitions and fuzzy transform, Soft Computing 21(13)(2017), 3479-3492.

[13] Močkoř, J., Holčapek, M., Fuzzy objects in spaces with fuzzy partitions, Soft Computing $\mathbf{2 1}(24)(2017), 72697284$.

[14] Močkoř, J., Monads and a Common Framework for Fuzzy type Automata, submitted

[15] V. Novák and I. Perfilijeva and J. Močkoř, Mathematical principles of fuzzy logic, Kluwer Academic Publishers, Boston, Dordrecht, London, 1999 .

[16] Perfilieva, I., Singh, A.P., Tiwari, S.P., On the relationships among F-transform, fuzzy rough sets and fuzzy topology, Soft Computing 21(13)(2017), 3513-3523.

[17] Perfilieva, I., Fuzzy transforms: theory and applications, Fuzzy Sets and Systems 157(2006), 9931023.

[18] Perfilieva, I., Singh, A.P., Tiwari, S.P., On the relationship among $F$-transform, fuzzy rough set and fuzzy topology, Proceedings of IFSAEUSFLAT, Amsterdam, Atlantis Press, 2015. s. 1324-1330.

[19] Rodabaugh, S.E., Powerset operator foundation for poslat fuzzy set theories and topologies, Höhle,U., Rodabaugh, S.E. (Eds.), Mathematics of Fuzzy Sets: Logic, Topology and Measure Theory, The Hnadbook of Fuzzy Sets Series, Vol. 3 ,
Kluwer Academic Publishers, Boston, Dordrecht (1999), 91-116.

[20] Rodabaugh, S.E., Powerset operator based foundation for point-set lattice theoretic (poslat) fuzzy set theories and topologies, Quaestiones Mathematicae 20(3)(1997), 463-530.

[21] Rodabaugh, S.E., Relationship of Algebraic Theories to Powerset Theories and Fuzzy Topological Theories for Lattice-Valued Mathematics, International Journal of Mathermatics and Mathematical Sciences 2007, 1-71.

[22] Solovyov, S. A., On the Category Set(JCPos), Fuzzy Sets and Systems 157(2006), 459-465.

[23] Solovyov, S. A., Categories of Lattice-Valued Sets as Categories of Arrows, Fuzzy Sets and Systems 157(2006), 843-854.

[24] Solovyov, S.A., Powerset operator foundations for catalg fuzzy set theories, Iranian Journal of Fuzzy Systems 8(2)(2001), 1-46.

[25] Stout, L. N., Topoi and Categories of Fuzzy Sets, Fuzzy Sets and Systems 12(1984), 169-184.

[26] Stout, L. N., A Survey of Fuzzy Sets and Topos Theory, Fuzzy Sets and Systems 42(1991), 3-14.

[27] Stout, L. N., Topoi and categories of fuzzy sets, Fuzzy Sets and Systems 12(2)(1984), 169-184.

[28] Wang, C.Y., Fuzzy rough sets based on generalized residuated lattices, Inf. Sci. 248(2013), 3149 .

[29] Wu, W.Z., Leung, Y., Mi, J.S., On characterization of $(\mathcal{J}, \mathcal{T})$-fuzzy rough approximation operators, Fuzzy Sets and Systems 154(2005), 76-102.

[30] L.A. Zadeh, Fuzzy sets, Information and Control 8(1965), 338-353. 